\title{
New biomarkers sought for improving sepsis management and care
}

For something so deadly, sepsis is surprisingly difficult to characterize. The body's response to a severe blood infection can be virtually identical to its response to other noninfectious triggers such as trauma. And even though approximately one-quarter of all people with severe sepsis eventually die from the disease, it's not easy for physicians to tell who the sickest ones are.

"You'd really like to be able to identify [severe sepsis] before it's obvious," says Mitchell Levy, medical director of the intensive care unit (ICU) at the Rhode Island Hospital in Providence. Right now, he points out, "you have to wait until organs fail until you can really tell how sick someone is."

To overcome this problem, researchers are hunting for new biomarkers that could be used to diagnose the condition earlier and single out individuals who are most likely to benefit from aggressive treatment. To date, however, even the best validated biomarkers can't differentiate sepsis caused by infections from other inflammatory causes and conditions.

Take procalcitronin (PCT), a precursor to a hormone involved in calcium metabolism that Frank $\mathrm{Gu}$, a bioengineer who studies sepsis biomarkers at the University of Waterloo in Ontario, describes as "the champion so far" when it comes to identifying bacterial infections. PCT levels in the blood typically jump 1,000-fold within hours of severe sepsis or septic shock setting in. However, concentrations of the peptide also spike after major trauma, elective surgery, severe burns and even some forms of cancer, which means it might not be specific enough to serve as a diagnostic marker of infection-caused sepsis.

That doesn't mean PCT can't be useful in the critical-care setting, however. In a study published online in May, Czech researchers reported that PCT levels were significantly higher in patients with sepsis infected with Gram-negative bacteria than in patients with either Gram-positive bacterial or fungal infections (Clin. Exp. Med. doi:10.1007/s10238$012-0191-8,2012)$. This finding suggests that the biomarker could help doctors narrow down appropriate treatment options. Still, most experts in the field agree that a more specific indicator of at-risk patients is needed.

\section{Parsing patients}

The 'soluble triggering receptor expressed on myeloid cells', sTREM-1, is one such candidate. In a study published last month, a Korean team measured plasma levels of sTREM-1 in 63 men with severe sepsis and found them to be significantly higher in individuals who ended up dying than in those who survived (Shock 37, 574-578, 2012).

A similar contender is suPAR (short for soluble urokinase-type plasminogen activator receptor). This protein is expressed on the surface of immune cells including neutrophils and macrophages and can differentiate dying patients from those who have both sepsis and pneumonia but will survive (J. Infect. 63, 344-350, 2011). In a 750-person observational trial, Austrian physicians are now evaluating whether measuring levels of suPAR and another biomarker shown to predict mortality in severe sepsis called ST2, a receptor involved in helper $\mathrm{T}$ cell responses, can help forecast not only clinical outcome but also infection type and treatment response.

Ultimately, this kind of biomarker combination approach will probably be more effective than any single biomarker would be. A team from the French University Hospital Centers in Nancy and Dijon has developed a 'Bioscore' that pools levels of three biomarkers: PCT, sTREM-1 and a type of glycoprotein expressed on the surface of neutrophils. Reporting in April, the French team showed that the composite metric performed better than each of the individual biomarkers-a finding that they validated in two separate cohorts (Am. J. Respir. Crit. Care Med. doi:10.1164/rccm.201201-0037OC, 2012).

The hope now, says Francois Philippart, an immunologist at the Pasteur Institute in Paris who is developing his own combination index, is to move these kinds of analysis out of clinical trials and into routine practice. "If we find an interesting panel of biomarkers, it will be useful for [diagnosing sepsis in] any kind of ICU patient," he says.

Melinda Wenner Moyer before they receive treatment. The failure to account for patient heterogeneity, Walker says, "has been the major mistake that big pharma has made."

\section{Pinning down the moving target}

Other researchers have turned their attention to inherited susceptibility to sepsis. For instance, a team from St. Paul's Hospital in Vancouver, Canada has been analyzing blood samples from patients with sepsis to look for mutations associated with disease risk. In a study published last year, they showed that people with a particular mutation in both copies of the gene encoding interleukin-17A, a cytokine involved in defending against microbial infection, were twice as likely to be infected with Gram-positive bacteria and about $50 \%$ more likely to die at 28 days compared to those with other gene variants (Crit. Care 15, R254, 2011).

Given this variance, a single medicine might produce varying results in a clinical trial if all these cases are grouped togethercreating a headache for drug developers and regulators alike. " $\mathrm{A}$ small subset of patients might really benefit and do so consistently, but this group could get lost in a large megatrial," says infectious disease specialist Steven Opal of the Brown University School of Medicine in Providence, Rhode Island. Some experts point out, for example, that patients with sepsis fare better if their illness stems from pneumonia in the lungs rather than from an infection in the abdomen. Pulling this group out into a separate trial could give a clearer picture of a particular drug's efficacy.

At the end of the day, however, Opal still thinks the traditional, catch-all approach might be best. "I personally like megatrials, as they are a good measure of effectiveness under real-world conditions," he says. Still, he concedes that there's plenty of room for improvement. One idea floated by experts at a two-day conference held in May at the US National Institutes of Health in Bethesda, Maryland, was to create a number of so-called 'Centers of Excellence'-institutions that are familiar with the problems associated with clinical trials in sepsis and could help raise the standard of current trial design and execution.

Angus would welcome such a move. "We would be better off spending our time enrolling a lot more patients into far larger randomized trials and getting to a much more robust answer much sooner," he says. "We still fundamentally believe in the idea that randomized trials help us make inferences of causality, but the vast majority of patients who get severe sepsis are never enrolled in clinical trials."

Roxanne Khamsi 